\title{
Substance Use End Relative to Reference Period
}

National Cancer Institute

\section{Source}

National Cancer Institute. Substance Use End Relative to Reference Period. NCI

Thesaurus. Code C83421.

A characterization of the relationship of a substance use conclusion point in time to a reference point in time. 